\section{Hiperlordose lombar em crianças e adolescentes de uma escola privada no Sul do Brasil: ocorrência e fatores associados}

\author{
Lumbar hyperlordosis in children and adolescents \\ at a privative school in southern Brazil: \\ occurrence and associated factors
}

\author{
1 Programa de Pós-graduação \\ em Ciências do Movimento \\ Humano, Universidade \\ Federal do Rio Grande do Sul, \\ Porto Alegre, Brasil. \\ Correspondência \\ A. T. Lemos \\ Programa de Pós-graduação \\ em Ciências do Movimento \\ Humano, Universidade Federal \\ do Rio Grande do Sul. \\ Rua Felizardo 750, Porto \\ Alegre, RS 90690-200, Brasil. \\ adrixlemos@gmail.com
}

\begin{abstract}
Posture assessment during childhood and adolescence allows early detection of postural disorders and adoption of preventive measures. This study evaluated the occurrence of lumbar hyperlordosis and associated factors in 467 children and adolescents aged 10 to 16 years at a school in Porto Alegre, Rio Grande do Sul State, Brazil. Lumbar hyperlordosis was defined as increased lumbar curvature evaluated by photography. Prevalence of lumbar hyperlordosis was 78\%. Factors positively associated with lumbar hyperlordosis were female gender ( $P R=1.08$; 95\%CI: 1.03; 1.13), abdominal strength below the 20th percentile $(P R=1.10 ; 95 \% C I: 1.05 ; 1.15)$, and flexibility below the 20th percentile $(P R=1.07 ; 95 \% C I$ : 1.01 ; 1.12). Lumbar hyperlordois was negatively associated with lumbar mobility $(P R=0.90 ; 95 \% C I$ : $0.85 ; 0.96)$ and height ( $P R=0.995 ; 95 \% C I$ : 0.99; 0.999). Effective strategies to improve physical fitness in schoolchildren are necessary, because low abdominal strength and low flexibility were associated with lumbar hyperlordosis.
\end{abstract}

Lordosis; Posture; Risk Factors
Adriana Torres de Lemos 1

Fábio Rosa dos Santos 1

Adroaldo Cezar Araujo Gaya 1

\section{Introdução}

O estudo da postura corporal e de suas relações com a aptidão física osteomuscular em crianças e jovens pode fornecer subsídios importantes para os profissionais da área da saúde, além de possibilitar uma melhor estruturação de seus programas de exercício. A investigação da ocorrência de alterações posturais, bem como das variáveis que podem se relacionar a essas condições, permite a detecção precoce de possíveis alterações e a adoção de estratégias preventivas. Nas fases da infância e da adolescência, ocorre a aceleração do crescimento e, sabendo-se que as variações de postura estão também associadas aos estágios de crescimento, esses períodos são importantes para intervenções e diminuição das condições predisponentes ao aparecimento dos problemas posturais. Estudos têm demonstrado a elevada prevalência de desvios posturais em crianças e jovens, principalmente da coluna lombar, entretanto a maioria das investigações não apresenta fatores associados, limitando-se à apresentação das prevalências 1,2,3,4,5.

Níveis satisfatórios de aptidão física relacionada à saúde são associados a menores riscos de desenvolvimento de doenças e/ou de condições crônico-degenerativas 6 . No entanto, carecemos de estudos que norteiem a atuação dos profissionais da saúde, principalmente no ambiente escolar, em relação aos níveis mínimos necessários de aptidão física que previnam a ocorrência de tais 
problemas. A flexibilidade e a força/resistência abdominal, quando em níveis adequados, são relacionadas à prevenção de alterações posturais e de dor lombar; relacionam-se, ainda, a um menor risco de lesões ligamentares 6,7. Diferentemente da aptidão cardiorrespiratória e do índice de massa corporal (IMC), para cuja avaliação já há pontos de corte, definidos a partir de estudos com escolares brasileiros 8,9,10,11,12, os níveis de aptidão física necessários para um menor risco de doenças osteomusculares e alterações posturais ainda não estão bem definidos. Além disso, muitos estudos ainda se utilizam de parâmetros internacionais, oriundos de escalas normativas, para avaliação da aptidão física relacionada à saúde de crianças e jovens brasileiros. Alguns estudos já vêm recomendando que as avaliações e interpretações de dados brasileiros sejam realizadas com cautela quando comparadas a dados internacionais 13 . Assim, torna-se necessário verificar se há associação entre alterações posturais e níveis de aptidão física relacionada à saúde, além de definir, em caso afirmativo, qual seria o critério recomendado para uma menor probabilidade dessas ocorrências.

A investigação de outros fatores associados também se faz necessária, tendo em vista a escassez de publicações sobre o tema. Em pesquisa prévia com escolares de 10 a 16 anos 14, idade, sexo e IMC, entre outros, não se associaram à presença de alteração postural.

Portanto, os objetivos deste estudo são verificar a ocorrência de hiperlordose lombar em crianças e adolescentes, além de identificar as variáveis associadas.

\section{Materiais e métodos}

Este estudo de caso de delineamento transversal foi realizado com escolares de 10 a 16 anos de idade, dos dois sexos, matriculados em uma escola privada da cidade de Porto Alegre, Rio Grande do Sul, Brasil. Todos os estudantes da faixa etária em estudo foram convidados a participar, sendo a amostra constituída por 467 crianças e adolescentes. Todas as avaliações foram realizadas durante as aulas de Educação Física por um grupo de profissionais previamente treinados.

Para a avaliação postural, foram marcados pontos de referência 15 com etiquetas adesivas e utilizado um simetrógrafo. As curvaturas cervical, dorsal e lombar foram avaliadas por meio de fotografias no plano sagital e de acordo com o sugerido por Kendall et al. 15. Os escolares estavam vestidos com roupas de banho e/ou short e top. As curvaturas foram classificadas em normais, aumentadas (hiperlordose cervical e lombar e hi- percifose dorsal) ou diminuídas (retificação cervical, dorsal e lombar). A objetividade das avaliações posturais foi testada pelo índice de concordância da estatística kappa em um subgrupo de 42 escolares, selecionados aleatoriamente em cada sexo e idade. Verificou-se excelente concordância intra $(0,834)$ e inter $(0,836)$ observador.

As componentes da aptidão física avaliadas foram força/resistência abdominal, verificada pelo teste abdominal em um minuto (sit up's), e flexibilidade, medida pelo teste de sentar e alcançar (sit and reach), ambas de acordo com as recomendações do Projeto Esporte Brasil (PROESP-BR). Testamos os percentis usualmente referidos na literatura para categorização da aptidão física (P10, P20, P25, P40, P60 e P80); seus respectivos valores foram considerados como pontos de cortes a partir dos quais classificávamos o escolar em "acima" ou "abaixo" deste determinado valor. $\mathrm{O}$ valor que melhor apresentou poder de discriminação para a variável desfecho foi o correspondente ao P20 da amostra deste estudo. Deste modo, os resultados obtidos nesses dois testes foram categorizados em acima ou abaixo do P20. Os valores do P20 para cada sexo e idade estão disponíveis no site do PROESP-BR (http://www.proesp.ufrgs.br). A mobilidade lombar foi avaliada pelo teste modificado de Schober 16, e seu resultado foi considerado "normal" ou "diminuído" quando maior ou menor que cinco centímetros, respectivamente.

$\mathrm{O}$ peso e a estatura foram medidos, e o IMC foi determinado por meio do cálculo da razão entre a medida de massa corporal em quilogramas e a estatura em metros elevada ao quadrado $\left[\mathrm{IMC}=\right.$ massa $(\mathrm{kg}) /$ estatura $\left.^{2}(\mathrm{~m})\right]$. Para a categorização do IMC, utilizamos os critérios de Conde \& Monteiro 17.

Quanto aos testes musculares, avaliou-se o encurtamento dos músculos isquiotibiais (EMI) e o encurtamento dos músculos flexores de quadril (EMFQ) conforme as orientações de Chaitow ${ }^{18}$. Os resultados dos testes foram categorizados em encurtamento ausente (flexão de quadril $\geq 90^{\circ}$, para EMI, e flexão de quadril $=0^{\circ}+$ flexão de joelho $\geq 80^{\circ}$, para EMFQ) ou encurtamento presente (flexão de quadril $<90^{\circ}$, para EMI, e flexão de quadril $>0^{\circ}+$ flexão de joelho $\geq$ ou $<80^{\circ}$; flexão de quadril $=0^{\circ}$ e flexão de joelho $<80^{\circ}$, para EMFQ).

Para a descrição das variáveis, utilizamos as frequências de ocorrência em valores percentuais, estratificados por sexo. Após análise bivariada, foi verificada a associação entre a ocorrência de hiperlordose lombar e as variáveis de aptidão física (força/resistência abdominal e flexibilidade), massa corporal, estatura, IMC, sexo, idade, mobilidade da coluna lombar, encurtamento 
de flexores de quadril e de isquiotibiais, além de postura da coluna dorsal e cervical, por meio da regressão de Poisson.

A hiperlordose lombar foi considerada como desfecho. As variáveis independentes foram idade (em anos completos), sexo (masculino - referência; feminino), mobilidade da coluna lombar (normal - referência; diminuída), força/ resistência abdominal (abaixo P20; acima P20 referência), flexibilidade (abaixo P20; acima P20 - critério), estatura, peso, IMC (normal - referência; excesso de peso; obesidade), encurtamento de isquiotibiais (ausente - referência; presente), encurtamento de flexores de quadril (ausente referência; presente), postura da coluna dorsal (neutra - referência; hipercifose; retificada) e da coluna cervical (neutra - referência; hiperlordose; retificada).

O projeto do estudo foi aprovado pelo Comitê de Ética da Universidade Federal do Rio Grande do Sul. Os pais ou responsáveis assinaram um Termo de Consentimento Livre e Esclarecido, concordando com a participação dos escolares no estudo.

\section{Resultados}

Dos 467 escolares participantes do estudo, 260 $(55,7 \%)$ eram do sexo masculino. As características antropométricas da amostra, estratificadas por sexo e idade, estão descritas na Tabela 1.

A hiperlordose lombar acometeu um total de $78,2 \%$ da amostra estudada. As meninas apresentam maior ocorrência ( $\mathrm{n}=175 ; 84,5 \%$ ) do que os meninos ( $\mathrm{n}=190 ; 73,1 \%)$. A associação entre o sexo feminino e a patologia foi estatisticamente significativa $\left(\chi^{2}=8,873 ; \mathrm{p}=0,005\right)$.

A Figura 1 apresenta a frequência de ocorrência de aumento da curvatura lombar ao longo das idades. Podemos observar que, independente do sexo, em todas as idades a frequência é superior a 50\%. Houve associação entre a idade e hiperlordose lombar no sexo masculino $\left(\chi^{2}=18,531\right.$; $\mathrm{p}=0,005)$.

Para melhor compreender as relações entre as variáveis, a partir do estabelecimento de um modelo conceitual baseado na revisão da literatura e considerando as variáveis de relevância biológica e clínica, recorremos à regressão de Poisson.

A Tabela 2 apresenta a análise da associação entre hiperlordose lombar e as variáveis independentes. Na análise bivariada, a patologia mostrou-se positivamente associada ao sexo feminino (RP = 1,07; IC95\%: 1,02; 1,11), idade de $10(\mathrm{RP}=1,10$; IC95\%: 1,08; 1,22) e 11 anos (RP = 1,11; IC95\%: 1,03; 1,19) e à força/resistência abdominal (RP = 1,09; IC95\%: 1,05; 1,14). Ob- servamos associação inversa com mobilidade lombar ( $\mathrm{RP}=0,94$; IC95\%: 0,87; 0,99), postura cervical retificada $(\mathrm{RP}=0,93$; IC95\%: 0,87; 0,99) e estatura (RP = 0,996; IC95\%: 0,994; 0,998). Após o ajuste para todas as outras variáveis do estudo, apenas sexo feminino, força/resistência abdominal, mobilidade lombar diminuída e estatura permaneceram associados à hiperlordose lombar. Por outro lado, a flexibilidade, que na análise bivariada não apresentou associação, passou a ter significância na etapa múltipla $(\mathrm{RP}=1,07$; IC95\%: 1,01; 1,12).

\section{Discussão}

Este estudo demonstrou elevados percentuais de hiperlordose lombar tanto nas meninas, quanto nos meninos. A prevalência preocupante dessa alteração entre jovens já vem sendo reportada por outros autores. Utilizando-se da mesma técnica de avaliação postural 15 adotada pela presente investigação, outros estudos também têm encontrado elevadas ocorrências da patologia em amostras com idades semelhantes 1,4. Campos et al. 19 , ao avaliarem a postura de 46 escolares de 9 a 18 anos de idade com sobrepeso e obesidade, encontraram $79 \%$ das meninas e $61 \%$ dos meninos com hiperlordose lombar. Outro estudo 5 , com amostra de 131 crianças de 10 a 13 anos do Município de General Câmara, no Rio Grande do Sul, detectou 65\% de jovens com aumento da curvatura lombar. Detsch et al. 20 verificaram a ocorrência de $66 \%$ de alterações posturais no plano sagital, em uma amostra de 495 estudantes de 14 a 18 anos da cidade de São Leopoldo, Rio Grande do Sul.

A diminuição nos níveis de aptidão física, em especial da força muscular no entorno da coluna lombar, seria uma possível causa para o aumento na lordose lombar. Murata et al. 21 verificaram, em estudo secular, aumento da curvatura lombar de jovens e a diminuição da força muscular de tronco. Segundo Magee 22 , a má postura é atribuída a fatores posicionais e estruturais. Dentre os fatores posicionais, o autor refere que, para a manutenção da postura correta, há a necessidade de músculos fortes, flexíveis e facilmente adaptáveis à alteração do ambiente. Nahas 6 também salienta que níveis satisfatórios de força muscular e de flexibilidade auxiliam na boa postura.

Os fatores associados à hiperlordose lombar foram sexo feminino, força/resistência abdominal, mobilidade lombar, estatura e flexibilidade. Dentre as variáveis da aptidão física relacionada à saúde, para a força/resistência abdominal, verificamos que a prevalência de hiperlordose lombar foi $10 \%$ mais elevada entre os escolares que não 
Características antropométricas de acordo com sexo e idade.

\begin{tabular}{|c|c|c|c|c|c|c|c|c|}
\hline \multirow[t]{3}{*}{ Idade (anos) } & \multicolumn{4}{|c|}{ Sexo masculino } & \multicolumn{4}{|c|}{ Sexo feminino } \\
\hline & $\mathbf{n}$ & Estatura & Massa & IMC & n & Estatura & Massa & IMC \\
\hline & & Média \pm DP & Média \pm DP & Média \pm DP & & Média \pm DP & Média \pm DP & Média $\pm \mathrm{DP}$ \\
\hline 10 & 20 & $145,8 \pm 1,5$ & $42,4 \pm 2,3$ & $19,7 \pm 0,8$ & 15 & $147,2 \pm 1,9$ & $40,9 \pm 1,7$ & $18,7 \pm 0,5$ \\
\hline 11 & 26 & $151,3 \pm 1,4$ & $46,8 \pm 2,0$ & $20,3 \pm 0,6$ & 25 & $154,2 \pm 1,2$ & $46,7 \pm 1,7$ & $19,5 \pm 0,6$ \\
\hline 12 & 42 & $158,3 \pm 1,3$ & $52,9 \pm 1,8$ & $20,9 \pm 0,6$ & 35 & $157,1 \pm 1,1$ & $50,1 \pm 1,6$ & $20,6 \pm 0,5$ \\
\hline 13 & 36 & $161,6 \pm 1,7$ & $54,7 \pm 2,2$ & $20,7 \pm 0,5$ & 21 & $158,5 \pm 1,5$ & $47,8 \pm 1,6$ & $18,9 \pm 0,5$ \\
\hline 14 & 44 & $169,9 \pm 1,0$ & $65,2 \pm 1,5$ & $22,6 \pm 0,5$ & 50 & $161,0 \pm 0,8$ & $52,7 \pm 0,8$ & $20,4 \pm 0,3$ \\
\hline 15 & 40 & $171,6 \pm 1,1$ & $63,5 \pm 2,1$ & $21,5 \pm 0,6$ & 29 & $161,3 \pm 0,9$ & $55,4 \pm 1,6$ & $21,3 \pm 0,6$ \\
\hline 16 & 52 & $174,3 \pm 0,9$ & $66,9 \pm 1,6$ & $21,1 \pm 0,5$ & 32 & $162,2 \pm 1,0$ & $54,5 \pm 1,1$ & $20,7 \pm 0,4$ \\
\hline
\end{tabular}

DP: desvio-padrão; IMC: índice de massa corporal.

Figura 1

Ocorrência de hiperlordose lombar, estratificada por sexo e idade.

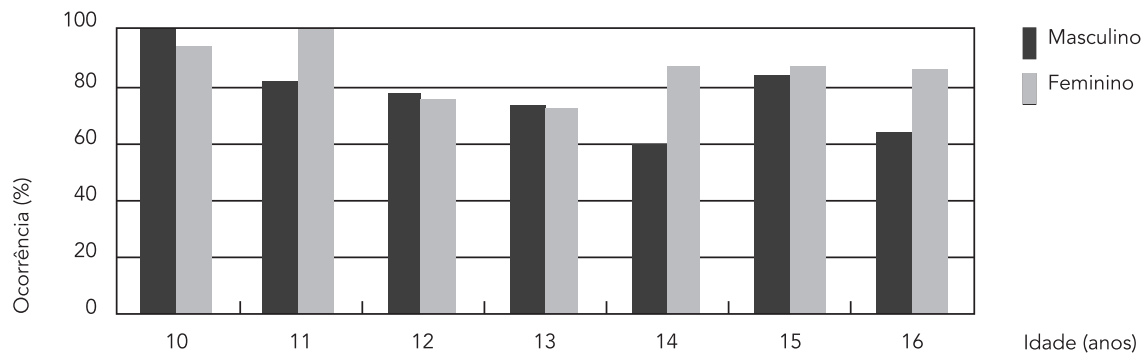

alcançaram o valor correspondente ao P20. Possivelmente isso se justifique pelo fato de os músculos abdominais exercerem importante função estabilizadora na coluna lombar 23 , auxiliando na manutenção da boa postura 6 . A pouca força desses músculos dificulta sua função de tração para cima sobre a pelve, permitindo que esta se incline para frente, aumentando a lordose 15. Estudo que verificou a força isométrica de flexores e extensores de tronco numa amostra de indivíduos com idades entre 13 e 60 anos concluiu que o desequilíbrio de forças entre os grupos musculares antagônicos influenciaram negativamente na curvatura lombar 24 . Esse resultado reitera a importância da prática de exercícios que sejam capazes, efetivamente, de melhorar a força/resistência abdominal.

Ainda referente à aptidão física relacionada à saúde, similar ao resultado encontrado para a força/resistência abdominal, a prevalência de hiperlordose lombar foi $7 \%$ mais elevada entre os escolares que se encontravam abaixo do P20 para a flexibilidade. Outros autores já mencionaram a importância desta variável na prevenção de problemas posturais 6,7,25. Os isquiotibiais, quando apresentam baixos níveis de flexibilidade, podem causar alteração do posicionamento sagital da pelve, gerando um desequilíbrio que pode levar ao aumento da curvatura lombar.

Houve associação entre hiperlordose lombar e o sexo feminino, sendo a prevalência $8 \%$ mais elevada nas meninas. Estudos demonstraram que o sexo feminino apresentou maiores ângulos de lordose lombar do que o masculino 26,27,28. Em adição, a investigação de Nguyen \& Schultz 29 verificou diferenças entre os sexos no alinhamento estático de membros inferiores (maior hiperextensão de joelhos nas mulheres) 
Fatores associados à hiperlordose lombar e razões de prevalência (RP) em crianças e adolescentes.

\begin{tabular}{|c|c|c|c|c|c|}
\hline \multirow[t]{2}{*}{ Variável } & \multirow[t]{2}{*}{$\%$} & \multicolumn{2}{|c|}{ Análise bivariada } & \multicolumn{2}{|c|}{ Análise múltipla } \\
\hline & & RP & IC95\% & RP & IC95\% \\
\hline \multicolumn{6}{|l|}{ Sexo } \\
\hline Feminino & 45,2 & 1,07 & 1,$02 ; 1,11$ & 1,08 & 1,$03 ; 1,13$ \\
\hline Masculino & 54,8 & 1,00 & Referência & 1,00 & Referência \\
\hline \multicolumn{6}{|l|}{ Idade (anos) } \\
\hline 10 & 7,7 & 1,15 & 1,$08 ; 1,22$ & 1,10 & 0,$99 ; 1,22$ \\
\hline 11 & 11,1 & 1,11 & 1,$03 ; 1,19$ & 1,07 & 0,$98 ; 1,18$ \\
\hline 12 & 16,2 & 1,02 & 0,$95 ; 1,10$ & 0,99 & 0,$90 ; 1,08$ \\
\hline 13 & 12,4 & 1,00 & 0,$92 ; 1,10$ & 0,97 & 0,$88 ; 1,06$ \\
\hline 14 & 20 & 1,00 & 0,$93 ; 1,09$ & 0,95 & 0,$88 ; 1,03$ \\
\hline 15 & 14,9 & 1,07 & 1,$00 ; 1,16$ & 1,03 & 0,$96 ; 1,12$ \\
\hline 16 & 17,5 & 1,00 & Referência & 1,00 & Referência \\
\hline \multicolumn{6}{|l|}{ Mobilidade lombar } \\
\hline Diminuída & 23,8 & 0,94 & 0,$87 ; 0,99$ & 0,90 & 0,$85 ; 0,96$ \\
\hline Normal & 76,2 & 1,00 & Referência & 1,00 & Referência \\
\hline \multicolumn{6}{|c|}{ Força/Resistência abdominal } \\
\hline Abaixo P20 & 14,4 & 1,09 & 1,$05 ; 1,14$ & 1,10 & 1,$05 ; 1,15$ \\
\hline Acima P20 & 85,6 & 1,00 & Referência & 1,00 & Referência \\
\hline \multicolumn{6}{|l|}{ Flexibilidade } \\
\hline Abaixo P20 & 16 & 1,04 & 0,$99 ; 1,09$ & 1,07 & 1,$01 ; 1,12$ \\
\hline Acima P20 & 84 & 1,00 & Referência & 1,00 & Referência \\
\hline \multicolumn{6}{|l|}{ IMC } \\
\hline Obesidade & 4,6 & 1,07 & 1,$00 ; 1,15$ & 0,95 & 0,$83 ; 1,09$ \\
\hline Excesso de peso & 33,5 & 1,00 & 0,$95 ; 1,04$ & 0,94 & 0,$88 ; 1,02$ \\
\hline Normal & 62 & 1,00 & Referência & 1,00 & Referência \\
\hline \multicolumn{6}{|c|}{ Encurtamento flexores quadril } \\
\hline Iliopsoas e reto & 24,5 & 1,03 & 0,$98 ; 1,08$ & 1,03 & 0,$98 ; 1,08$ \\
\hline Iliopsoas & 13,3 & 0,98 & 0,$91 ; 1,05$ & 0,97 & 0,$90 ; 1,03$ \\
\hline Reto femoral & 33 & 0,96 & 0,$90 ; 1,01$ & 0,98 & 0,$92 ; 1,03$ \\
\hline Sem encurtam. & 29,2 & 1,00 & Referência & 1,00 & Referência \\
\hline \multicolumn{6}{|c|}{ Encurtamento isquiotibiais } \\
\hline Presente & 48,7 & 1,02 & 0,$97 ; 1,06$ & 1,01 & 0,$97 ; 1,06$ \\
\hline Ausente & 51,3 & 1,00 & Referência & 1,00 & Referência \\
\hline \multicolumn{6}{|l|}{ Postura cervical } \\
\hline Retificada & 15,4 & 0,93 & 0,$87 ; 0,99$ & 0,94 & 0,$88 ; 1,01$ \\
\hline Hiperlordose & 31,3 & 1,01 & 0,$97 ; 1,06$ & 1,03 & 0,$99 ; 1,08$ \\
\hline Neutra & 53,3 & 1,00 & Referência & 1,00 & Referência \\
\hline \multicolumn{6}{|l|}{ Postura dorsal } \\
\hline Hipercifose & 49 & 0,99 & 0,$95 ; 1,04$ & 1,03 & 0,$98 ; 1,08$ \\
\hline Neutra & 51 & 1,00 & Referência & 1,00 & Referência \\
\hline Estatura $(\mathrm{cm})$ & & 0,996 & 0,$994 ; 0,998$ & 0,0995 & 0,$99 ; 0,999$ \\
\hline Peso $(\mathrm{kg})$ & & 0,998 & 0,$99 ; 1,00$ & 1,00 & 0,$99 ; 1,01$ \\
\hline
\end{tabular}

IC95\%: intervalo de 95\% de confiança; IMC: índice de massa corporal. 
e pelve (maior inclinação anterior nas mulheres) de estudantes com idade média de 21 anos. Em decorrência dessas diferenças entre os sexos, a avaliação da inclinação pélvica segue critérios diferenciados, sendo considerado normal, além do equilíbrio pélvico, o limite de um centímetro de inclinação anterior para as meninas e de um centímetro de inclinação posterior para os meninos 30 . No presente estudo, apesar de respeitados esses aspectos durante a avaliação, a prevalência de hiperlordose lombar foi elevada e, em virtude dessa predisposição do sexo feminino, faz-se ainda mais necessário o fortalecimento da musculatura estabilizadora da pelve.

A mobilidade lombar associou-se negativamente à hiperlordose lombar, ou seja, aqueles que pertenciam à categoria "mobilidade lombar diminuída”, apresentaram menor prevalência desta alteração postural $(R P=0,90)$, quando comparados aos que apresentaram mobilidade considerada normal. Esta variável foi também testada nas análises bivariada e múltipla de forma contínua e permaneceu atuando com comportamento similar; logo, a cada aumento em uma unidade de centímetro, houve aumento na razão de prevalência de hiperlordose lombar. Tendo em vista que parte do sistema de estabilização da coluna vertebral é fornecido pelos ligamentos, é possível que menor mobilidade limite maiores angulações da curvatura lombar.

Apresentando o mesmo comportamento da mobilidade lombar, a estatura também se associou negativamente à hiperlordose lombar. Para cada aumento de um centímetro na estatura, houve uma chance reduzida na ocorrência da alteração $(\mathrm{RP}=0,995)$. Vindo ao encontro desse resultado, Martelli \& Traebert 14 verificaram que escolares com estatura abaixo de 1,54 metros apresentaram maior ocorrência de hiperlordose lombar do que os com estatura superior a esse ponto de corte (dicotomizados em abaixo e acima da média de estatura). As razões para essa ocorrência precisam ser mais bem investigadas.

Quanto à variável idade, na análise bivariada, aos 10 e 11 anos, as prevalências de hiperlordose lombar eram superiores em 15\% e $11 \%$, respectivamente, quando comparadas às prevalências aos 16 anos de idade. Essa relação não se manteve na análise múltipla, na qual a idade passou a não ter associação significativa. Contudo, quando estratificamos por sexo, houve associação entre hiperlordose lombar e idade para os meninos. Na Figura 1, verificamos que não há uma diminuição nos percentuais de ocorrência da patologia dos 10 aos 14 anos de idade, voltando a aumentar aos 15 e 16 anos. No sexo feminino, em todas as idades, a prevalência é superior a $80 \%$, exceto aos 12 e 13 anos, fase em que as frequências são mais baixas.

O presente estudo verificou a ocorrência de hiperlordose lombar em escolares de uma escola privada de Porto Alegre. Por se configurar como um estudo de caso, os achados desta investigação podem ser extrapolados apenas para escolares que apresentem características similares às da amostra estudada. Carecemos de pesquisas com escolares brasileiros as quais apontem os fatores associados à ocorrência dessa alteração postural; considerando que a maioria se detém na demonstração de sua prevalência 4,5,19,20,31. Assim, entendemos que, a partir de estudos de casos, seja possível verificar semelhanças e diferenças entre os fatores associados à ocorrência de hiperlordose lombar em escolares de diversas regiões, proporcionando melhor entendimento dessa patologia.

A amostra estudada apresentou elevada ocorrência de hiperlordose lombar, sendo esta observada em $78 \%$ dos adolescentes avaliados. Em todas as idades estudadas, independente do sexo, a ocorrência do aumento da curvatura lombar foi maior do que $50 \%$. Os fatores positivamente associados a essa alteração foram sexo, força/resistência abdominal e flexibilidade. Por outro lado, mobilidade lombar e estatura se associaram negativamente à hiperlordose lombar.

Uma vez que se assuma que determinados níveis de aptidão física relacionada à saúde se associam com menor risco de dores e problemas osteomusculares, a avaliação e o acompanhamento, bem como o desenvolvimento dessas variáveis, devem ser realizados na infância e adolescência no ambiente escolar, durante as aulas de Educação Física. Assim, é importante ressaltar a necessidade de implementação de programas efetivos de promoção da saúde nas escolas, visando à obtenção/manutenção de níveis satisfatórios de aptidão física.

Em síntese, a prevalência de hiperlordose lombar encontrada é preocupante e indica a necessidade de que as variáveis modificáveis que estão associadas a esta condição sejam contempladas em programas de educação para a promoção da saúde. 


\section{Resumo}

A avaliação da postura corporal durante a infância e adolescência permite a detecção de alterações em fases iniciais e a adoção de medidas preventivas. Avaliouse a ocorrência de hiperlordose lombar e fatores associados em 467 crianças e adolescentes de 10 a 16 anos de idade de uma escola de Porto Alegre, Rio Grande do Sul, Brasil. A hiperlordose lombar foi definida como aumento da curvatura lombar avaliada por meio de fotografia. Verificou-se 78\% de ocorrência da patologia. Os fatores positivamente associados a essa alteração postural foram sexo feminino $(R P=1,08$; IC95\%: 1,03; 1,13), força/resistência abdominal abaixo do percentil 20 (RP = 1,10; IC95\%: 1,05; 1,15) e flexibilidade abaixo do percentil 20 ( $R P=1,07$; IC95\%: 1,01; 1,12). A hiperlordose lombar se associou negativamente à mobilidade lombar ( $R P=0,90$; IC95\%: 0,85; 0,96) e à estatura (RP = 0,995; IC95\%: 0,99; 0,999). Estratégias efetivas que melhorem os níveis de aptidão física de escolares são necessárias, tendo em vista que baixos níveis de força e flexibilidade foram associados à hiperlordose lombar.

Lordose; Postura; Fatores de Risco

\section{Colaboradores}

A. T. Lemos participou da concepção do projeto de pesquisa, coleta, análise e interpretação dos dados e redação do artigo. F. R. Santos contribuiu na interpretação dos dados e redação do artigo final. A. C. A. Gaya foi orientador na concepção e planejamento do projeto de pesquisa e na interpretação dos dados; colaborou na revisão crítica do conteúdo e aprovação da versão final do manuscrito.

\section{Agradecimentos}

Ao Conselho Nacional de Desenvolvimento Científico e Tecnológico (CNPq) pela bolsa de produtividade científica (PQ1D-CNPq)

\section{Referências}

1. Detsch C, Candotti CT. A incidência de desvios posturais em meninas de 6 a 17 anos da cidade de Novo Hamburgo. Movimento (Porto Alegre) 2001; 7:43-56.

2. Machado DT. Incidência de alterações posturais da coluna lombar e joelhos e sua relação com a flexibilidade de tronco e força de abdominais em estudantes entre 10 e 13 anos de idade [Monografia]. Porto Alegre: Rede Metodista de Educação do Sul; 2003.

3. Ribeiro CZP, Akashi PMH, Sacco ICN, Pedrinelli A. Relação entre alterações posturais e lesões do aparelho locomotor em atletas de futebol de salão. Rev Bras Med Esporte 2003; 9:91-7.

4. Penha PJ, João SMA, Casarotto, RA, Amino CJ, Penteado DC. Postural assessment of girls between 7 as 10 years of age. Clinics 2005; 60:9-16.

5. Lemos AT, Machado DT, Moreira R, Torres L, Garlipp DC, Lorenzi TDC, et al. Atitude postural de escolares de 10 a 13 anos de idade. Revista Perfil (UFRGS) 2005; 7:53-9.
6. Nahas MV. Atividade física, saúde e qualidade de vida: conceitos e sugestões para um estilo de vida ativo. 3a Ed. Londrina: Midiograf; 2003.

7. Kapandji IA. Fisiologia articular: tronco e coluna vertebral. São Paulo: Editora Manole; 1990.

8. Guedes DP, Guedes JERP, Barbosa DS, Oliveira JA. Aptidão física relacionada à saúde e fatores de risco predisponentes às doenças cardiovasculares em adolescentes. Rev Port Ciênc Desporto 2002; 2:31-46.

9. Lunardi CC, Petroski EL. Índice de massa corporal, circunferência da cintura e dobra cutânea triciptal na predição de alterações lipídicas em crianças com 11 anos de idade. Arq Bras Endocrinol Metab 2008; 52:1009-14.

10. Bergmann GG. Aptidão física relacionada à saúde cardiovascular: proposição de pontos de corte para escolares brasileiros [Tese de Doutorado]. Porto Alegre: Universidade Federal do Rio Grande do Sul; 2008. 
11. Moreira RB. Composição corporal de escolares de 10 a 15 anos: um estudo longitudinal [Dissertação de Mestrado]. Porto Alegre: Universidade Federal do Rio Grande do Sul; 2009.

12. Silva MF. Promoção da Saúde: a relação entre aptidão física e fatores de risco biológico das doenças cardiovasculares em escolares [Tese de Doutorado]. Porto Alegre: Universidade Federal do Rio Grande do Sul; 2009.

13. Bergmann GG, Araújo MLB, Garlipp DC, Lorenzi TDC, Gaya A. Alteração anual no crescimento e na aptidão física relacionada à saúde de escolares. Rev Bras Cineantropom Desempenho hum 2005; 7:55-61.

14. Martelli RC, Traebert J. Estudo descritivo das alterações posturais da coluna vertebral em escolares de 10 a 16 anos de idade - Tangará, SC, 2004. Rev Bras Epidemiol 2006; 9:87-93.

15. Kendall FP, McCreary EK, Provance PG. Músculos, provas e funções. 4a Ed. São Paulo: Editora Manole; 1995.

16. Macrae I, Wright V. Measurement of back movement. Ann Rheum Dis 1969; 28:584-9.

17. Conde WL, Monteiro CA. Body mass index cutoff points for evaluation of nutritional status in Brazilian children and adolescents. J Pediatr (Rio J.) 2006; 82:266-72.

18. Chaitow L. Técnicas de palpação: avaliação e diagnóstico pelo toque. São Paulo: Editora Manole; 2001.

19. Campos F, Silva A, Fisberg M. Descrição fisioterapêutica das alterações posturais de adolescentes obesos. http://www.brazilpednews.org.br/ju nh2002/obesos.pdf (acessado em 08/Nov/2006).

20. Detsch C, Luz AMH, Tarragô CC, Oliveira DS, Lazaron F, Guimarães LK, et al. Prevalência de alterações posturais em escolares do ensino médio em uma cidade no Sul do Brasil. Rev Panam Salud Pública $2007 ; 21: 231-8$.
21. Murata Y, Utsumi T, Hanaoka E, Takahashi K, Yamagata M, Moriya H. Changes in lumbar lordosis in young patients with low back pain during a 10-year period. J Orthop Sci 2002; 7:618-22.

22. Magee DJ. Avaliação músculo-esquelético. 3a Ed São Paulo: Editora Manole; 2002.

23. Konin J. Cinesiologia prática para fisioterapeutas. Rio de Janeiro: Editora Guanabara Koogan; 2006.

24. Kim HJ, Chung S, Kim S, Shin H, Lee J, Kim S, et al. Influences of trunk muscles on lumbar lordosis and sacral angle. Eur Spine J 2006; 15:409-14.

25. Whithead E, Corbin CB. Aptidão muscular. Horizonte: Revista de Educação Física e Desporto 1986; 8:136-41.

26. Youdas JW, Hollman JH, Krause DA. The effects of gender, age, and body mass index on standing lumbar curvature in persons without current low back pain. Physiother Theory Pract 2006; 22:229-37.

27. Norton BJ, Sahrmann SA, van Dillen FL. Differences in measurements of lumbar curvature related to gender and low back pain. J Orthop Sports Phys Ther 2004; 34:524-34.

28. Fernand R, Fox DE. Evaluation of lumbar lordosis: a prospective and retrospective study. Spine 1985; 10:799-803.

29. Nguyen AD, Shultz SJ. Sex differences in clinical measures of lower extremity alignment. J Orthop Sports Phys Ther 2007; 37:389-98.

30. Santos A. Diagnóstico clínico postural: um guia prático. São Paulo: Editora Summus; 2001.

31. Santos CIS, Cunha ABN, Braga VP, Saad IAB, Ribeiro MAGO, Conti PBM, et al. Ocorrência de desvios posturais em escolares do ensino público fundamental de Jaguariúna, São Paulo. Rev Paul Pediatr 2009; 27:74-80.

Recebido em 29/Jul/2011

Versão final reapresentada em 19/Out/2011 Aprovado em 30/Nov/2011 